\title{
Semi-Supervised learning with Collaborative Bagged Multi-label K-Nearest-Neighbors
}

https://doi.org/10.1515/comp-2019-0017

Received May 17, 2019; accepted August 29, 2019

\begin{abstract}
Over the last few years, Multi-label classification has received significant attention from researchers to solve many issues in many fields. The manual annotation of available datasets is time-consuming and need a huge effort from the expert, especially for Multi-label applications in which each example of learning is associated with many labels at once. To overcome the manual annotation drawback, and to take advantages from the large amounts of unlabeled data, many semi-supervised approaches were proposed in the literature to give more sophisticated and fast solutions to support the automatic labeling of the unlabeled data. In this paper, a Collaborative Bagged Multi-label K-Nearest-Neighbors (CobMLKNN) algorithm is proposed, that extend the co-Training paradigm by a Multi-label K-Nearest-Neighbors algorithm. Experiments on ten real-world Multi-label datasets show the effectiveness of CobMLKNN algorithm to improve the performance of $M L K N N$ to learn from a small number of labeled samples by exploiting unlabeled samples.
\end{abstract}

Keywords: Semi supervised learning, Collaborative Bagging, Multi-label classification, Multi-label K-NearestNeighbors.

\section{Introduction}

Machine learning includes two main approaches called: generative models and discriminative models, the $\mathrm{K}$ Nearest Neighbors classifier belongs to the second category that aims to learn the connection between an observation (described by a set of attributes) and the targeted

\footnotetext{
*Corresponding Author: Nesma Settouti: Biomedical Engineering Laboratory, Faculty of Technology, Tlemcen University, 13000 Tlemcen, Algeria, E-mail: nesma.settouti@univ-tlemcen.dz Khalida Douibi, Mohammed El Amine Bechar, Mostafa El Habib Daho, Meryem Saidi: Biomedical Engineering Laboratory, Faculty of Technology, Tlemcen University, 13000 Tlemcen, Algeria
}

class in a given dataset. Several scenarios are possible, in supervised learning, the class is already annotated by the expert for each example in the initial learning set, the algorithm search for the optimal function to automatically assign the correct labels to each instance.

However, in unsupervised learning, the observations are unlabeled. Indeed, the algorithm must search for the corresponding labels based on several strategies, as Clustering that aims to discover the inherent groupings in data, the similarity between data points is computed according to their given attributes. Another type of learning form is known as semi-supervised, in which labels are only known for a small number of observations that will be used to annotate unlabeled data, the process of clustering is mainly used also for this task.

The present work focuses on semi-supervised approaches to learn from Multi-labeled data. This task was greatly applied by researchers to annotate images, for example, a picture taken by a user and posted on social media could include many labels simultaneously as: "selfie", "child", "summer", "hashlabels" etc.

The presence of multiple labels for the same example may increase significantly the complexity of the labeling task. To deal with that, many approaches were recently proposed to face the major challenges of the modern Multi-label data analysis applications, since it is complex and time consuming compared to single-label annotation in which the human annotator must identify only one class to label an instance, whereas, in the Multi-label case, every possible label for each instance should be considered. Semi-supervised learning was highly used by researchers to reduce labeling efforts and produce more instances for the training process of the algorithms, especially, for Multi-label classification.

The semi-supervised learning approaches proposed in the literature for single label problems have shown their efficiency, however, they are not directly applicable to the Multi-label case. The adaptation of such methods to the Multi-label learning is possible using two strategies [1], either by modifying the existing single label algorithms to fit data (Adaptation Algorithms) or by transforming the 
Multi-label data to fit the algorithm (Problem Transformation). Finally, the strategy of learning using a committee of learners was also applied in this field by using Ensemble methods from the first and/or the second family of approaches.

In this work, we discussed the benefits of applying semi-supervised Multi-label learning approaches to improve the performance of one of the most studied Multilabel algorithms, that adapts K-Nearest-Neighbors rule to the Multi-label framework, known as MLKNN [2]. A collaborative Bagging approach is proposed, which extend the co-Training paradigm [3] based on ensemble method.

Our proposed method is named CobMLKNN (Collaborative Bagged Multi-label K-Nearest-Neighbors), it consists of firstly building a Bagging of Multi-label K-NearestNeighbors $(M L K N N)$ based on the labeled training set. Then, the algorithm improves the performance of the ensemble of classifiers by exploiting unlabeled samples to enrich the learning set. At each iteration, each MLKNN relearn based on the newly labeled by its concomitant ensemble, only labeled example with a confidence level exceeds a threshold are selected.

The main contributions of this paper are summarized as follows.

1. First, the semi-supervised approach was considered for Multi-label K-Nearest-Neighbors algorithm,

2. Second, an extension of collaborative Bagging approach [4] to Multi-label framework was proposed.

The rest of this paper is organized as follows. Section 2 provides an overview of some relevant research achievements obtained with ensemble method strategy in supervised and semi-supervised Multi-label learning. Section 3 describes the proposed approach CobMLKNN in details. Section 4 reports the experimental results and corresponding analysis followed by the conclusions in Section 5 .

\section{Related Work}

Recently, several approaches have been developed to solve Multi-label learning problems [5, 6]. This section aims to overview some well-known methods for supervised and semi-supervised learning.

Multi-label learning has received increasing attention in the past decade. Initially, it has been applied for text categorization applications [7]; then, it has been expanded to resolve a variety of issues such as multi-media content classification including images [8-12], audio [13-15], videos [16-19], Web and rule mining [20-25], tag recom- mendation [26, 27], information search [28-30], bioinformatics [31-34] etc.

In the literature, Multi-label algorithms were categorized into two main categories [1]: Transformation algorithms and Adaptation algorithms. The former transforms the data to fit the algorithm; however, the later modify the algorithm to fit the data. In addition, Ensemble methods were widely used by Multi-label community researchers to resolve many issues in this field, as dealing with the problem of high complexity of Label Powerset (LP) approach [35], and to solve the problem of not considering Label dependence by the learner in the case of Binary Relevance (BR) models [36]. Binary Relevance and Label Powerset are two main transformations applied by researchers to deal with Multi-label problem [37, 38], the first one transforms the Multi-label Learning problem to several Binary Classification problems and learns for each label a separate classifier. However, the second one considers each new combination of labels existing in the learning set as a new class, i.e Multi-label Learning problem is transformed into a Multi-class Classification problem. Similarly, several researchers have exploited Ensemble methods for enhancing the generalization of Multi-label algorithms in both categories. We review in the following paragraphs some works based on Ensemble methods from Adaptation and transformation methods.

In [39], the authors proposed an ensemble Multi-label classifier based on Label Powerset strategy to resolve the multi-disease risk prediction based on physical examination records. Another work that aims to reduce the problem of complexity of Label Powerset based Ensemble of Pruned Sets (EPS) was proposed in [40], the idea was to samples with rare label sets to allow the model focusing on the important label combinations, the bagging was applied as a strategy of learning of EPS. For Binary Relevance approaches, many works were proposed as Ensemble Classifier Chain ECC [41] that use an ensemble of Classifier Chain, each one learns random Chain orderings using a random subset of training instances. Similarly, Ensemble of Binary Relevance classifiers (EBR) was proposed in [36], where the base classifier here is a Binary Relevance instead of Classifier Chain.

For the adaptation category, Ensemble methods was applied for many algorithms as in [1], in which Random Forest [42] was adapted to Multi-label Learning using MLC4.5 as base model, called: RFMLC4.5. In [43], the authors developed Multi-label Ensemble Learning (EnML) based evolutionary algorithms to optimize each classifier in the ensemble and to find the optimal labels for each instance. 
One of the major limitations of Supervised Multi-label learning is need of labeled datasets to learn accurately [44]. Therefore, the development of semi-supervised algorithms is growing nowadays to face this issue and help to extract information from unlabeled datasets.

Many works were developed based on classical semisupervised learning methods, as co-training [45, 46], transductive [47-49], and graph-based [50, 51] semisupervised algorithms. Among them, the authors in[52] proposed the SSWL (Semi-Supervised Weak-Label) method for labeling multi-label data, instance similarity and label similarity were considered to supplant missing labels. The ensemble of multiple models is used to improve the robustness when label information is insufficient. Based on the co-training strategy which works under inductive setting, [46] proposed a novel approach COINS (CO-training for INductive Semi-supervised Multilabel learning) to learn from labeled and unlabeled data by augmenting the diversity between the two classifiers on each feature subset. For text classification, the authors in [53] have implemented a semi-supervised Multi-label model for jointly achieving document and sentence-level class inferences. For image classification, a Multi-label Semi-Supervised Active Image Classification (ML-SSAIC) approach was proposed by [54] to handle the problem of high labors costs caused by manual annotation in supervised Multi-label learning. Moreover, an adaptive semi-supervised learning strategy to train a Multi-label classifier was adapted by [55], it learns an intermediate features space for both labeled and unlabeled training samples via low-rank matrix recovery.

Several works that study feature selection approach for semi-supervised Multi-label learning was proposed. In [56], the authors formulate the Multi-label Learning as an Ensemble learning problem to improve both Classification and feature selection tasks, they proposed a new semisupervised Multi-label feature selection approach based on the ensemble paradigm. Furthermore, [57] proposed a space consistency-based feature selection model to extract label correlation information with incomplete prior knowledge and embed this information in feature selection. This mechanism contributes to properly extracting label information in a semi-supervised Multi-label learning scenario and adequately employing this information to select discriminative features. Similarly, a framework for semi-supervised multi-label classification was proposed by [58], that exploit specific features of the label set based on a semi-supervised clustering to extract useful information of both labeled and unlabeled instances together.

In this paper, a Collaborative Bagged Multi-label KNearest-Neighbors (CobMLKNN) algorithm is proposed, the idea is to improve the learning of each Multi-label KNearest-Neighbors classifier in the Bagging with the newly labeled instances by its concomitant learners, that means we extend the co-Training paradigm to Multi-label Ensemble method. The CobMLKNN improve the performance of each classifier in the Bagging, that learns initially from a small number of labeled samples by exploiting confident newly labeled samples.

\section{Proposed Method}

The present section details the idea of Semi-Supervised Multi-label Classification based Collaborative Bagged Multi-label K-Nearest-Neighbors algorithm. The goal of Semi-supervised learning is to construct a better classifier by exploring a large amount of unlabeled data. First, we review briefly the Bagged Multi-label K-Nearest-Neighbors (BagMLKNN) proposed in [59], then we explain the idea of extending the Co-training paradigm to a Multi-label framework for BagMLKNN and its main advantages.

\subsection{Bagged Multi-label K-Nearest-Neighbors (BagMLKNN)}

Initially, the Bagging was introduced with a basic rule, a Decision Tree [60]. Then, many researchers extended the concept to other basic rules such as Nearest Neighbor rule in order to benefit from its major advantages, including its simplicity to implement, its easy adaptability to any learning methods and the fact that it reduces the impact of the choice of the training set on classification results. For those reasons, the BagMLKNN was previously proposed in [59] to improve the performance of one of the well-known Multi-label algorithm called MLKNN [2].

$M L K N N$ adapts the $K N N$ to the Multi-label scenario by using a posteriori principle to determine the label set of unseen instance [2], the algorithm follows two major steps for building an initial model. As illustrated in Figure 1, in the first step, the a priori probabilities for each label are calculated, then, the conditional probabilities for each label are computed as the proportion of instances with the considered label i.e the K-Nearest-Neighbors who share the same label.

For a new instance, MLKNN search for K-NearestNeighbors by measuring the similarity between the new instance and all the examples of the dataset using the Euclidean distance, then, the presence of each label in the Neighborhood is used as evidence to compute Maximum $A$ 


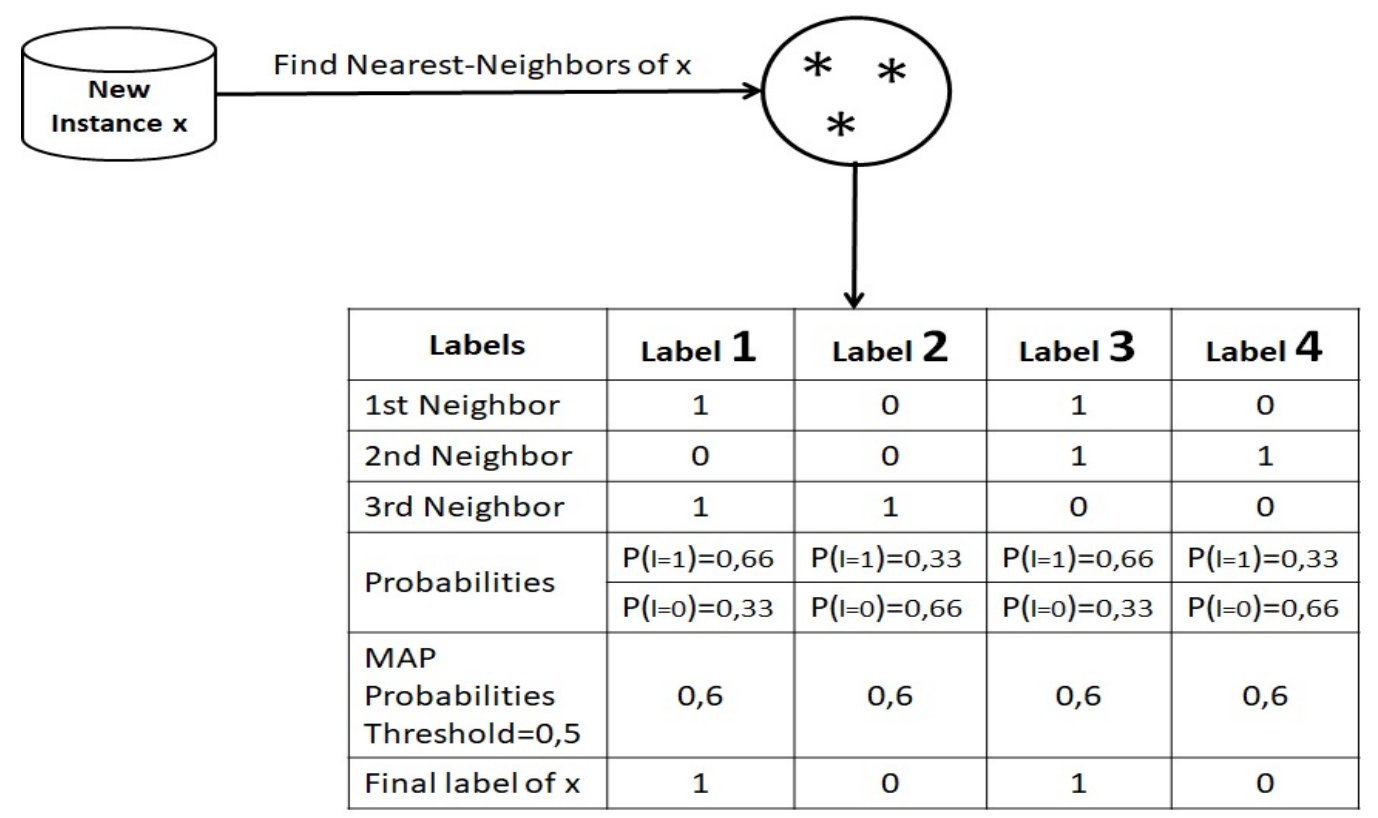

Figure 1: $M L K N N$ principle example to classify a new instance.

Posteriori (MAP) probabilities from the Conditional ones obtained before [6]. The label set of the new sample is generated from the MAP probabilities and the probability itself is provided as a confidence level for each label, thus making possible to also generate a label Ranking [6].

The BagMLKNN method as illustrated in Figure 2 resume the idea of using several $M L K N N$ simultaneously for the classification process. In fact, the benefits of Ensemble methods starting from a basic rule relatively poor (Rule of $M L K N N$ that is not consistent), the Bagging turn it into a very good rule with asymptotic properties (consistency and optimal convergence rate). In addition, Bagging has many advantages [60] because it is simple to implement, easily adapts to any learning methods and reduces the impact of the choice of the training set on Classification results.

The satisfactory results obtained by using several $M L K N N$ simultaneously for the prediction of labels in BagMLKNN [59] conducted us to extend the approach to semi-supervised learning framework in order to take advantage from unlabeled datasets. Indeed, the availability of unlabeled data and the difficulty of the manual annotation, make the semi-supervised strategies a required and important task. In the present work, we focus on improving the performance of a supervised Multi-label algorithm by exploring unlabeled instances in the dataset by using the Co-training paradigm.

The idea behind the semi-supervised learning is to handle simultaneously with few labeled instances and a large number of unlabeled instances while getting bene- fit from the information provided by unlabeled data. In such learning configuration, the key assumption is that two examples will be assigned to similar labels if they overlap in their input space. In this case, the MLKNN algorithm is an excellent candidate, its principle allows to identify the $\mathrm{K}$ nearest instances of each test instance and counts the number of neighbors belonging with the same label. Then, it uses the Maximum A posteriori Principle (MAP) to determine the label set for each test instance. Besides, ensemble multi-label models are efficient and computationally practical approaches. The Multi-label KNearest-Neighbors based Bagging approach BagMLKNN has proven its efficiency, therefore, in this work, we apply semi-supervised learning for a bagging $M L K N N$ via coTraining and co-Forest with an extension of the confidence measure to the multi-label context.

\subsection{Collaborative Bagged Multi-label K-Nearest-Neighbors (CobMLKNN)}

Co-training algorithm [3] is one of the most attractive semisupervised ensemble models, where two base-learner are initially trained using two redundant and independent sets of features. In further iterations, each base-learner classifies the unlabeled examples, adds the most confident examples in the training set. As an improvement of the coTraining algorithm, Lee and Zhou proposed the Co-forest approach [4]. In this method, an ensemble of diverse baseclassifiers is used instead of redundant and independent 


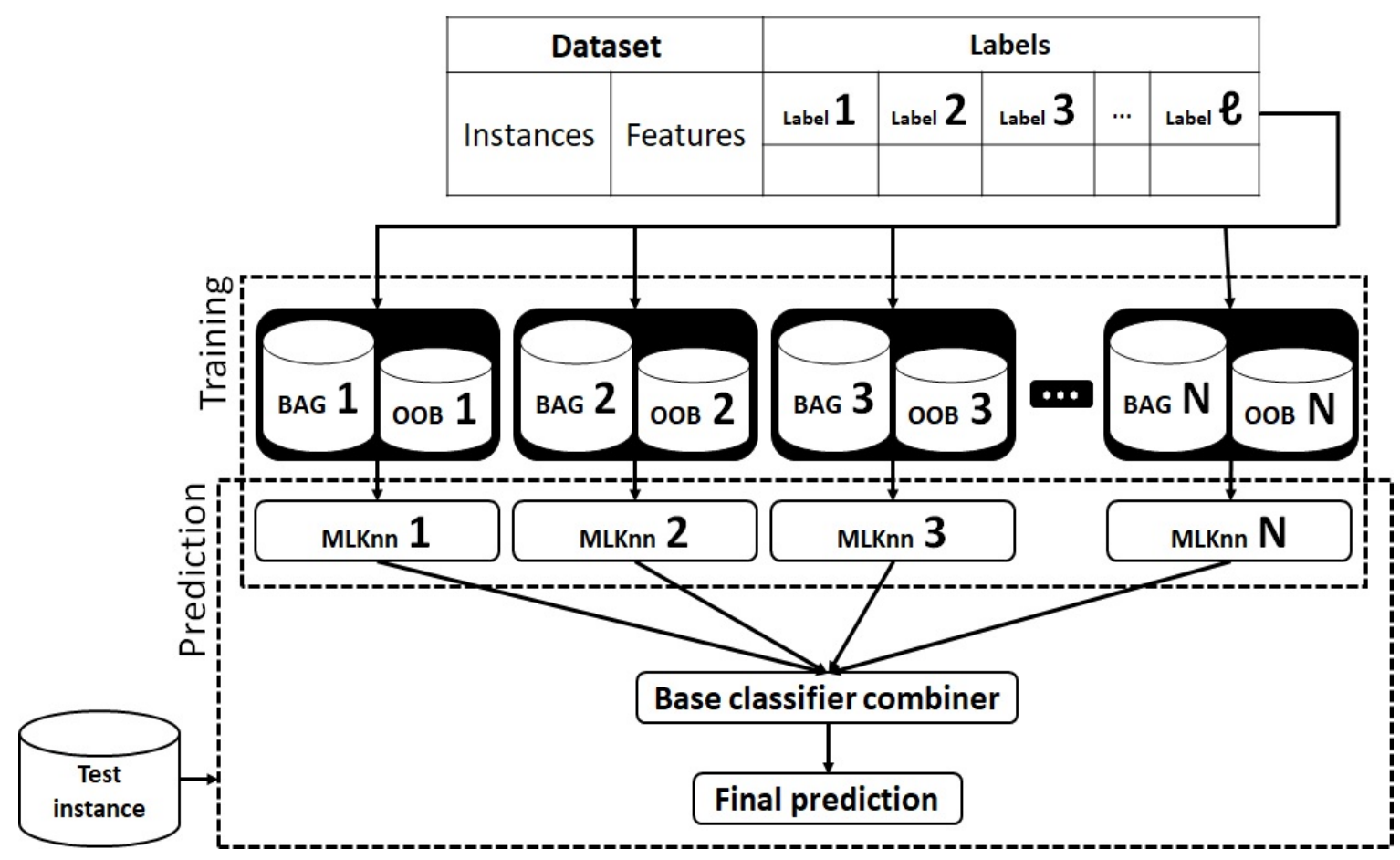

Figure 2: Classification process with BagMLKNN

views. The committee of diverse accurate classifiers is initially constructed by using a bagging strategy. At each iteration and for each classifier, a subset of unlabeled examples is drawn randomly from the whole unlabeled data set and classified using the concomitant ensemble [61]. The most confident examples to label are then determined and the committee members are retrained using their updated training sets.

The CobMLKNN algorithm extends the co-Forest [3] paradigm to Multi-label K-Nearest-Neighbors based Bagging approach known as: BagMLKNN. The basic idea of CobMLKNN is to form a set of MLKNN classifiers using the labeled set denoted by $L$. Then, each classifier is relearned on new instances labeled by its concomitant ensemble from the unlabeled set denoted by $U$. Our notation follows the same standards proposed in [4, 37], where a Multi-label data set is denoted $D$ with $q$ instances. For each example $(x i, Y i), i=1, \ldots, q, x_{i}$ is the set of attributes values and $Y_{i}$ is the set of true labels, each one belonging to the set of $\ell$ labels $=$ Label $_{j}: j=1, \ldots, \ell$. Let $H$ be the set of $N M L K N N$ classifiers. $H^{\star}$ is the concomitant ensemble of $h_{i}$ i.e. all classifiers except $h_{i}$ with $(i=1, \ldots, N-1)$ $H^{\star}=H_{N-1}$.

In our method (Algorithm 1), we apply the same principle used in Co-forest, to first build a set of $M L K N N$ classifiers on labeled $L$ data (Algorithm 1 line 1) and then refine each classifier with unlabeled ones by its concomitant ensemble. The confidence of the labeling is computed as the degree of agreement on the labeling, i.e. the number of classifiers that agree on the label assigned by each $h_{i}$ of $H^{\star}$. In this way, we can effectively estimate the confidence of each $M L K N N$ classifier in the ensemble.

In several semi-supervised learning algorithms, a problem resides in the principle of adding the unlabeled data of which confidence is above $\theta$ to $L_{i}$, which make $L_{i}$ very bulky. Except, in the case where a classifier cannot represent the underlying distribution; a huge amount of labeled data becomes detrimental to performance, instead of improving the prediction accuracy.

To overcome this problem, similarly to the Co-forest [4] algorithm, the CobMLKNN incorporates the principle of assigning a weight to each unlabeled example in an iterative manner as proposed by Nigam et al. [62]. A sample is weighted according to the predictive confidence of its concomitant classifiers. In the $t-1$ iteration $((t-1)$ with $t>1$, a condition (1) for updating the training set should be satisfied to iteratively improve the generalization ability. This approach reduces the impact of $\theta$, even if it is small, the examples with low predictive confidence will be reduced. 
Algorithm 1. CobMLKNN pseudo code

Input: $L$ : Labeled dataset,

$U$ : Unlabeled dataset,

$\theta$ : confidence threshold,

$K$ : number of Nearest-Neighbors,

$N$ : number of MLKNN,

$\ell$ : number of Labels.

Output: BagMLKNN H,

Processus:

1: Build BagMLKNN $H$ on $(L, \ell)$.

2: Initialization :

$\hat{e}_{i, 0} \leftarrow 0.5$,

$W_{i, 0} \leftarrow 0$

$t \leftarrow 0$

3: Repeat until there is no change in the MLKNN.

4: $t \leftarrow t+1$

5: for $i=1 \rightarrow N$ do

$\hat{e}_{i, t} \leftarrow$ EstimateError $(H, L, \ell)$

$L_{i, t} \leftarrow \phi$

if $\left(\hat{e}_{i, t}<\hat{e}_{i, t-1}\right)$ then

$U_{i, t} \leftarrow$ Subsample $\left(\mathrm{U}, \frac{\hat{e}_{i, t-1} \cdot W_{i, t-1}}{\hat{e}_{i, t}}\right)$

for $x_{u, \ell} \in U_{i, t}$ do

if Confidence $\left(H^{\star}, x_{u, \ell}, \ell\right)>\theta$ then

$L_{i, t}^{\prime} \leftarrow L_{i, t} \cup\left(x_{u, \ell}, H^{*}\left(x_{u, \ell}\right)\right)$

$W_{i, t} \leftarrow W_{i, t}+$ Confidence $\left(H^{\star}, x_{u, \ell}, \ell\right)$

end if

end for

end if

end for

for $i=1 \rightarrow N$ do

if $\left(\hat{e}_{i, t} \cdot W_{i, t}<\hat{e}_{i, t-1} \cdot W_{i, t-1}\right)$ then

$h_{i} \leftarrow \operatorname{LearnMLKNN}\left(L \bigcup\left(L_{i, t}^{\prime}\right)\right)$

end if

end for

Return $h_{i}$

Majority vote $H(x) \leftarrow \operatorname{argmax}_{\text {Label } \in \ell} \sum_{i: h_{i}(x)=y} 1$

$$
\frac{\hat{e}_{i, t}}{\hat{e}_{i, t-1}}<\frac{w_{i, t-1}}{w_{i, t}}<1
$$

- $h_{i}$ is the classifier of re-learning phase that learns from $L_{i} U L_{i, t}^{\prime}$ at $t$ i-th iteration.

- $\quad \hat{e}_{i, t}$ is the error rate of $H$ on $L_{i, t}^{\prime}$,

- $\hat{e}_{i, t} w_{i, t}$ is the average weight of the mislabeled examples by $H$.

The following assumptions: $\hat{e}_{i, t}<\hat{e}_{i, t-1}$ and $w_{i, t-1}<$ $w_{i, t}, w_{i, t}<\frac{\hat{e}_{i, t-1} w_{i, t-1}}{\hat{e}_{i, t}}$ should be satisfied at the same time. However, even if this requirement is respected, $\hat{e}_{i, t} W_{i, t}<$ $\hat{e}_{i, t-1} W_{i, t-1}$ might still be violated since $W_{i, t}$ might be much larger than $W_{i, t-1}$. In this case, to verify the equation (1), the new labeled data $L_{i, t}^{\prime}$ must be sub-sampled so that $W_{i, t}$ is less than $\frac{\hat{e}_{i, t-1} W_{i, t-1}}{\hat{e}_{i, t}}$.

In CobMLKNN Algorithm 1 lines (8-9), a subset of unlabeled examples with the total weight less than $\frac{\hat{e}_{i, t-1} W_{i, t-1}}{\hat{e}_{i, t}}$ is randomly selected from $U$. Then, confident examples are further selected from the subset (Algorithm 1 in lines 11-14 and Algorithm 2). Note that it not only offers diversity but also reduce the chance of being trapped in a local minimum, just like a similar strategy employed in co-Training and Co-forest.

More specifically, in CobMLKNN, for each unlabeled example $x_{u, \ell}$, each member $h_{i} \in H^{*}$, will annotate it with a vector of labels $\ell$, in order to generate the class probability distribution for the given $x_{u, \ell}$ for each Label in $\ell$. Then, a majority vote is applied over $H^{\star}$ for each $\ell$, in order to assign the final vector of labels of $x_{u, \ell}$.

\section{Algorithm 2. Confidence Function}

Input: Unlabeled example $x_{u, \ell}$, committee of classifiers $H^{\star}$ and number of Labels $\ell$

1: Apply $H^{\star}$ to generate the class probability distribution for each Label $\ell$ of $x_{u, \ell}$

2: for Label $=1 \rightarrow \ell$ do

3: $\quad$ V_label $\left(x_{u, \ell}\right)=\operatorname{argmax}_{1 \leq \text { Label } \leq \ell} \mathrm{P}\left(x_{u, \ell}\right)$

4: $\quad$ V_conf $\left(x_{u, \ell}\right)=\max _{1 \leq \text { Label } \leq \ell} \mathrm{P}\left(x_{u, \ell}\right)$

5: end for

6: return $\operatorname{conf}\left(x_{u, \ell}\right)=\frac{\sum V_{\_} \operatorname{conf}\left(x_{u, \ell}\right)}{\ell}$ and V_label $\left(x_{u, \ell}\right)$

As described in Algorithm 2, each classifier from $H^{\star}$ is asked to label $x_{u, \ell}$, in order to generate the class probability distribution for the given $x_{u, \ell}$. Then, the vector class which receives the maximal votes is assigned to the example $x_{u, \ell}$, with label confidence equal to the sum of the class probability distribution for each label over the total number of labels $\ell$. An illustrative example in Figure 3 , shows how the confidence measure procedure for an instance $x_{u, 4}$ assigns the four labels combination with a concomitant ensemble $H^{\star}$ equal to 5 .

More precisely, in each iteration of CobMLKNN, the concomitant ensemble $H^{\star}$ will test each example in $U$. For an unlabeled example $x_{u, \ell}$, if the number of classifiers that agree on a particular label exceeds a predefined threshold $\theta$, this new label is assigned to the example and then it will be added to the new set $L^{\prime}$ that will be used for refining $h_{i}$ in the next iterations (Algorithm 1 in lines 18-21).

In summary, the principle of the algorithm CobMLKNN (as illustrated in Figure 4) consists of $N$ Multi-label KNearest-Neighbors that are first built on a labeled boot- 


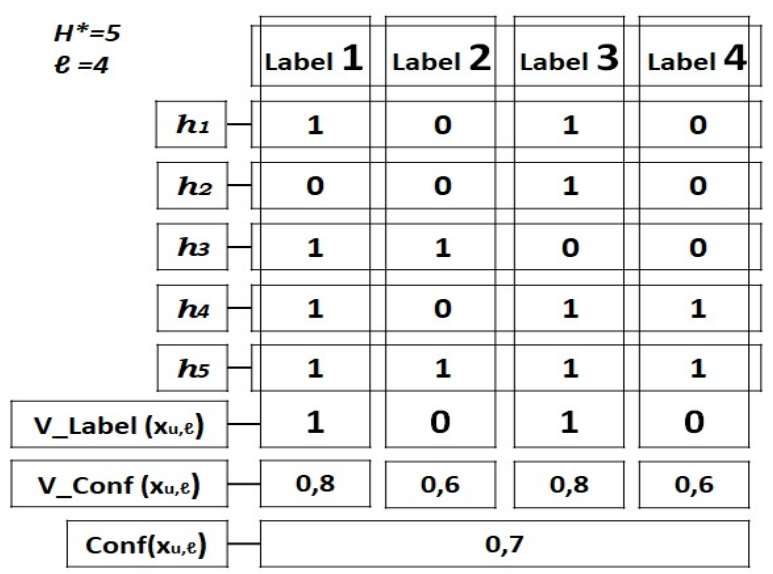

Figure 3: An illustrative example for the Confidence measure function.

strap training set $L$ to create a random forest. Then, at each iteration, each Multi-label K-Nearest-Neighbors classifier will be readjusted with new instances newly labeled by its concomitant ensemble, where the confidence of the labeled example exceeds a certain threshold of $\theta$.

\section{Experiments and Results}

In this section, the application framework with associated protocols are presented, the experiments were conducted on ten real-word multi-label datasets (Table 1) from the Mulan's repository [63] that were selected from different domains: two in biology (yeast, genbase), five in multimedia (emotions, scene, corel5k, mediamill and flags), three in text (enron, medical and bibtex).

In this section, the application framework with associated protocols are presented, the experiments were conducted on ten real-word Multi-label datasets (Table 1) from the Mulan's repository [63] that were selected from different domains: two in biology (yeast, genbase), five in multimedia (emotions, scene, corel5k, mediamill and flags), three in text (enron, medical and bibtex).

We indicate the label cardinality $\mathrm{L}_{C}$ and the label density $\mathrm{L}_{D}$ of each dataset, where $\mathrm{L}_{C}$ represents the average number of labels associated with each instance, while $\mathrm{L}_{D}$, indicates the average proportion of labels associated with each example.
Table 1: Description of the Multi-label data sets used in the experiments.

\begin{tabular}{l|ccccc}
\hline Dataset & \#Attribute & \#Instance & \#Label & $\mathrm{L}_{C}$ & $\mathrm{~L}_{D}$ \\
\hline Bibtex & 1836 & 7395 & 159 & 2.402 & 0.015 \\
Corel5k & 499 & 5000 & 374 & 3.522 & 0.009 \\
Enron & 1001 & 1702 & 53 & 3.378 & 0.064 \\
Emotions & 72 & 593 & 6 & 1.869 & 0.311 \\
Flags & 19 & 194 & 7 & 3.392 & 0.485 \\
Food & 21 & 407 & 12 & 2.28 & 0.19 \\
Genbase & 1186 & 662 & 27 & 3.522 & 0.009 \\
Medical & 1449 & 978 & 45 & 1.245 & 0.028 \\
Scene & 294 & 2407 & 6 & 1.074 & 0.179 \\
Yeast & 103 & 2417 & 14 & 4.237 & 0.303 \\
\hline
\end{tabular}

The performance of Multi-label algorithms depends not only on the algorithm itself but also on other parameters as the datasets used for the learning and the metrics used for its evaluation. The latter are divided into two groups: Bipartitions-based and Ranking-based:

- The Bipartitions-based assessment measures are calculated based on the comparison of the relevant predicted labels with the actual relevant labels. These assessment measures are based on the average differences between the actual and predicted label sets. In this work, we apply the measures: Hamming loss (2), accuracy (3), F-score (4) and subset accuracy (5).

$$
\begin{gathered}
\text { H_Loss } \left.=\frac{1}{q} \sum_{i=1}^{q}\left|Y_{i}\right| X O R\left|\hat{Y}_{i}\right|\right) \\
\text { Accuracy }=\frac{1}{q} \sum_{i=1}^{q} \frac{\left|Y_{i} \cap \hat{Y}_{i}\right|}{\left|Y_{i} \cup \hat{Y}_{i}\right|} \\
F-\text { score }=\frac{1}{q} \sum_{i=1}^{q} \frac{2\left|Y_{i} \cap \hat{Y}_{i}\right|}{\left|Y_{i}\right|+\left|\hat{Y}_{i}\right|} \\
\text { Sub_Acc }=\frac{1}{q} \sum_{i=1}^{q} I\left(Y_{i}=\hat{Y}_{i}\right),
\end{gathered}
$$

With $Y_{i}$ the sets of true labels for an instance, $\hat{Y}_{i}$ the sets of predicted labels for an instance and $q$ the total instances and $I$ is a function that maps a true logic proposition to 1 and false to 0 .

- Ranking-based assessment measures compare the predicted ranking of labels with the actual ranking of the Multi-label dataset. Overall, we use four rankingbased measures as presented in [37]: one-error (6), coverage (7), ranking loss (8) and average precision (9).

$$
O \_E r r o r=\frac{1}{q} \sum_{i=1}^{q} \delta\left(\operatorname{argmin}\left[r_{i}(\text { Label })\right]\right)
$$




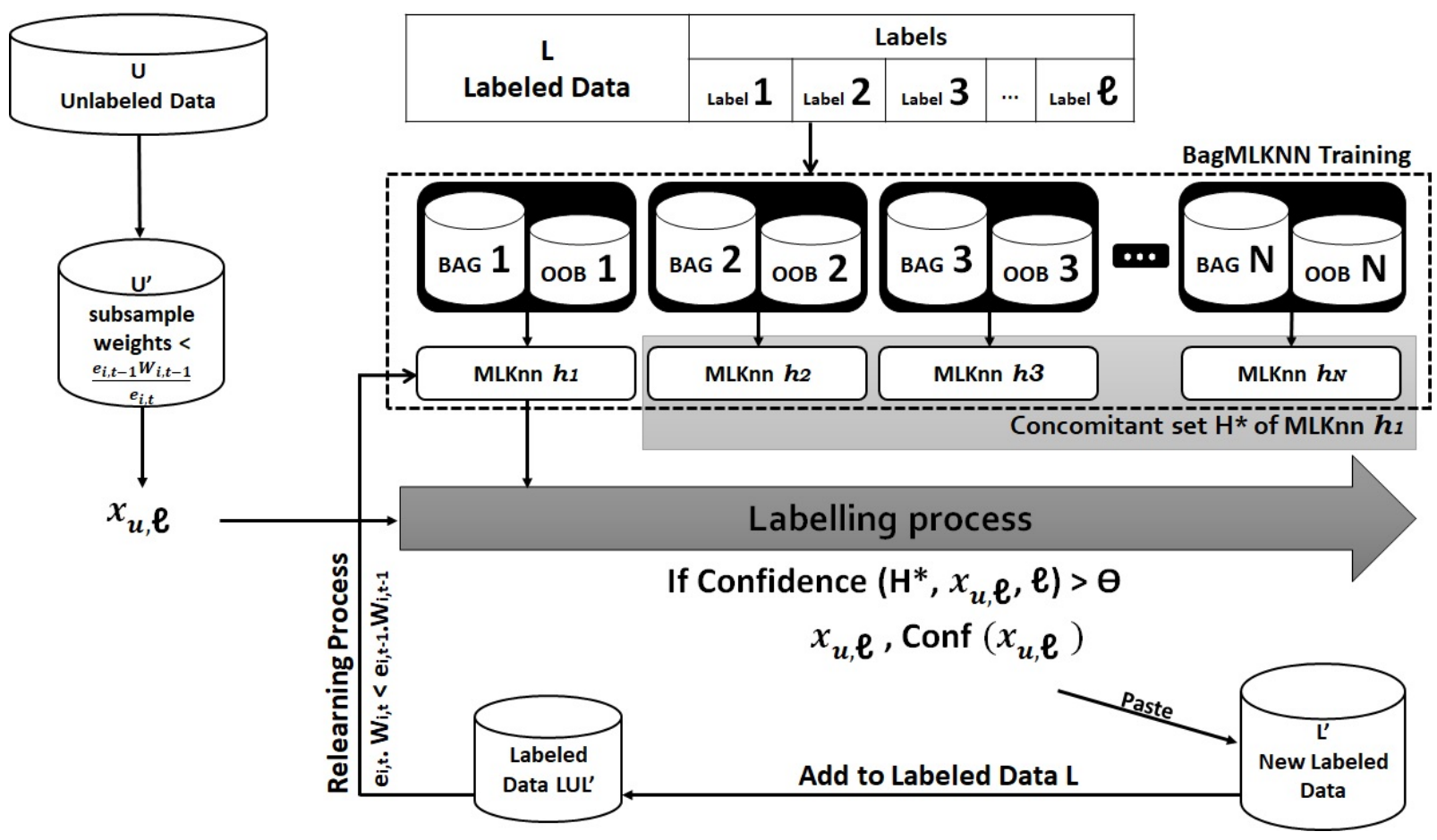

Figure 4: The CobMLKNN procedure.

where

$$
\text { Label } \in \ell, \delta(\text { Label }) \begin{cases}1 & \text { if Label } \notin Y_{i} \\ 0 & \text { otherwise }\end{cases}
$$

and $r_{i}($ Label $)$ is the predicted rank of class label Label $_{i}$ for a given instance.

$$
\operatorname{Cov}=\frac{1}{q} \sum_{i=1}^{q} \max \left[r_{i}(\text { Label })\right]-1
$$

Where Label $\in Y_{i}$.

$$
\begin{aligned}
& \text { Rank_Loss }=\frac{1}{q} \sum_{i=1}^{q} \frac{1}{\left|Y_{i}\right|\left|\overline{Y_{i}}\right|} \times \\
& \mid\left\{\left(\text { Label }_{a}, \text { Label }_{b}\right)\right\} \mid r_{i}\left(\text { Label }_{a}\right) \leq r_{i}\left(\text { Label }_{b}\right)
\end{aligned}
$$

with $\left(\right.$ Label $_{a}$, Label $\left._{b}\right) \in Y_{i} \times \overline{Y_{i}}$ and $\overline{Y_{i}}$ is the complementary set of $Y_{i}$ with respect to $\ell$.

$$
\begin{aligned}
& \text { Avg_Prec } \\
= & \frac{1}{q} \sum_{i=1}^{q} \frac{1}{\left|Y_{i}\right| \sum_{\text {Label }_{a} \in Y_{i}} \frac{\mid \text { Label }_{b} \in Y_{i}: r_{i}\left(\text { Label }_{b}\right) \leq r_{i}\left(\text { Label }_{a}\right) \mid}{r_{i}\left(\text { Label }_{a}\right)}}
\end{aligned}
$$

The algorithms was evaluated using 5-fold crossvalidation with a set of $N=6$ of $M L K N N$ classifiers (as used in Co-forest [4] algorithm) and the number of
Nearest-Neighbors $K$ fixed to 12 while keeping the same default value of the smoothing parameter=1. The training data are randomly divided into two sets: $L$ labeled and unlabeled $U$ determined by a rate $(\mu)$, which is calculated by the size of $L$ on the size of $L \cup U$. To simulate different amounts of labeled data, four different labeled rates $\mu=$ $10 \%, 30 \%, 40 \%$ and $80 \%$, are studied. Also, we note that the distributions of class in $L$ and $U$ are maintained similar to the original set.

In the following experiments, confidence level $\theta$ is set at 0.85 , i.e. a newly labeled example is considered as confident if at least five of the six $M L K N N$ classifiers agree on an assigned label. To estimate the performances on each dataset, we have predetermined a labeled rate of $\mu$ of labeled examples. The labeled samples were randomly selected, with the only constraint: the presence of at least one example of each class for each set. For each set, the algorithm is evaluated on its ability to correctly predict the labels of unlabeled examples.

In the following paragraphs, we discuss the results of experiments with each evaluation metric.

\section{Accuracy}

In the Multi-label domain, accuracy is defined as the proportion between the number of correctly predicted labels 
Table 2: Results under a labeled rate $\mu=10 \%$

\begin{tabular}{|c|c|c|c|c|c|c|c|c|c|}
\hline Datasets & Methods & $\operatorname{Acc} \uparrow$ & Sub_Acc $\uparrow$ & F-score $\uparrow$ & H_Loss $\downarrow$ & Avg_Prec $\uparrow$ & $\operatorname{Cov} \downarrow$ & O_Error $\downarrow$ & R_Loss $\downarrow$ \\
\hline \multirow[t]{3}{*}{ BIBTEX } & MLKNN & 0,9849 & 0,0017 & 0,0710 & 0,0151 & 0,1594 & 80,2249 & 0,8159 & 0,3713 \\
\hline & BagMLKNN & 0,9855 & 0,0094 & 0,0787 & 0,0148 & 0,1807 & 78,4720 & 0,7809 & 0,3554 \\
\hline & CobMLKNN & 0,9852 & 0,0105 & 0,0789 & 0,0145 & 0,1834 & 81,0982 & 0,8162 & 0,3464 \\
\hline \multirow[t]{3}{*}{ COREL5K } & MLKNN & 0,9903 & 0,0000 & 0,0344 & 0,0097 & 0,1730 & 173,2031 & 0,8314 & 0,2674 \\
\hline & BagMLKNN & 0,9908 & 0,0000 & 0,0000 & 0,0092 & 0,2064 & 156,9941 & 0,7827 & 0,2227 \\
\hline & CobMLKNN & 0,9908 & 0,0001 & 0,0074 & 0,0092 & 0,2082 & 156,0517 & 0,7866 & 0,2204 \\
\hline \multirow[t]{3}{*}{ ENRON } & MLKNN & 0,9373 & 0,0004 & 0,2537 & 0,0627 & 0,5083 & 17,1915 & 0,4586 & 0,1549 \\
\hline & BagMLKNN & 0,9371 & 0,0024 & 0,2632 & 0,0629 & 0,5032 & 18,4282 & 0,4392 & 0,1414 \\
\hline & CobMLKNN & 0,9377 & 0,0092 & 0,2887 & 0,0623 & 0,5055 & 17,4998 & 0,4225 & 0,1384 \\
\hline \multirow[t]{3}{*}{ EMOTIONS } & MLKNN & 0,5990 & 0,0495 & 0,3270 & 0,3921 & 0,5467 & 3,2939 & 0,6899 & 0,4604 \\
\hline & BagMLKNN & 0,6003 & 0,0626 & 0,3105 & 0,4010 & 0,5307 & 3,2687 & 0,6525 & 0,4566 \\
\hline & CobMLKNN & 0,6079 & 0,0687 & 0,3182 & 0,3937 & 0,5351 & 3,2434 & 0,6455 & 0,4527 \\
\hline \multirow[t]{3}{*}{ FLAGS } & MLKNN & 0,6246 & 0,0708 & 0,6295 & 0,3345 & 0,7784 & 4,0246 & 0,2828 & 0,3374 \\
\hline & BagMLKNN & 0,6620 & 0,0462 & 0,6854 & 0,3380 & 0,7838 & 3,9938 & 0,2473 & 0,2854 \\
\hline & CobMLKNN & 0,6655 & 0,0338 & 0,6425 & 0,3354 & 0,7969 & 4,2923 & 0,1411 & 0,2543 \\
\hline \multirow[t]{3}{*}{ FOOD } & MLKNN & 0,8110 & 0,1967 & 0,4151 & 0,1890 & 0,6736 & 4,4596 & 0,3364 & 0,3095 \\
\hline & BagMLKNN & 0,8206 & 0,1691 & 0,4020 & 0,1794 & 0,6554 & 4,4246 & 0,3805 & 0,2579 \\
\hline & CobMLKNN & 0,8393 & 0,2672 & 0,4233 & 0,1607 & 0,6924 & 4,4436 & 0,2941 & 0,2503 \\
\hline \multirow[t]{3}{*}{ GENEBASE } & MLKNN & 0,9510 & 0,0670 & 0,1048 & 0,0490 & 0,4604 & 6,7900 & 0,6706 & 0,2587 \\
\hline & BagMLKNN & 0,9585 & 0,2036 & 0,2805 & 0,0415 & 0,5874 & 8,0959 & 0,4588 & 0,3154 \\
\hline & CobMLKNN & 0,9636 & 0,2362 & 0,3595 & 0,0364 & 0,6710 & 6,0814 & 0,3710 & 0,2266 \\
\hline \multirow[t]{3}{*}{ MEDICAL } & MLKNN & 0,9723 & 0,0656 & 0,0963 & 0,0277 & 0,4290 & 8,5276 & 0,6816 & 0,2266 \\
\hline & BagMLKNN & 0,9702 & 0,0571 & 0,0996 & 0,0298 & 0,3883 & 11,9724 & 0,7264 & 0,3723 \\
\hline & CobMLKNN & 0,9725 & 0,0571 & 0,1248 & 0,0275 & 0,4387 & 8,4945 & 0,6656 & 0,2257 \\
\hline \multirow[t]{3}{*}{ SCENE } & MLKNN & 0,8299 & 0,1917 & 0,3175 & 0,1700 & 0,7101 & 0,9720 & 0,4775 & 0,1771 \\
\hline & BagMLKNN & 0,8209 & 0,2068 & 0,3660 & 0,1790 & 0,6127 & 0,9700 & 0,5433 & 0,1926 \\
\hline & CobMLKNN & 0,8432 & 0,2486 & 0,3853 & 0,1767 & 0,7407 & 0,8583 & 0,4324 & 0,1538 \\
\hline \multirow[t]{3}{*}{ YEAST } & MLKNN & 0,7454 & 0,0166 & 0,5262 & 0,2546 & 0,6749 & 7,4002 & 0,3119 & 0,2327 \\
\hline & BagMLKNN & 0,7616 & 0,0256 & 0,5332 & 0,2384 & 0,6965 & 7,1042 & 0,2888 & 0,2164 \\
\hline & CobMLKNN & 0,7704 & 0,0739 & 0,5555 & 0,2296 & 0,7087 & 6,9700 & 0,2747 & 0,2111 \\
\hline \multirow{3}{*}{$\begin{array}{l}\text { Averaged } \\
\text { Ranking }\end{array}$} & MLKNN & 2.7 & 2.45 & 2.5 & 2.2 & 2.3 & 2.4 & 2.6 & 2.7 \\
\hline & BagMLKNN & 2.1 & 2.2 & 2.2 & 2.45 & 2.5 & 2 & 2.1 & 2.3 \\
\hline & CobMLKNN & 1.2 & 1.35 & 1.3 & 1.35 & 1.2 & 1.6 & 1.3 & 1 \\
\hline
\end{tabular}

and the total number of active labels, both in the actual and predicted label sets. The measure is calculated for each instance and then averaged, like all metrics based on the example. Therefore, in the Multi-label classification, a low accuracy rate is not revealing that this model is less efficient. A prediction containing a subset of labels is better than a prediction that contains none, indeed, a prediction that succeeds in recognizing two of the three labels is preferable to a prediction without a label.

The results given in tables 2, 3, 4, 5 are very close in the case of high labeling threshold. However, it shows that our proposed method CobMLKNN achieves better rates with a low number of learning examples, due to the integration of the newly labeled instances with the use of the confidence metric to enrich the learning set.

\section{Subset Accuracy}

Subset Accuracy is the strictest metric, indicating the percentage of samples with all labels correctly predicted. For a consequence, the disadvantage of this measure is that ignore the partially correct predictions by the classifier. From tables 2, 3, 4, 5, we note that CobMLKNN outperforms the other supervised algorithms even for a dataset with a low number of labeled examples due to the involvement of unlabeled instances to improve the learning process. 
Table 3: Results under a labeled rate $\mu=30 \%$

\begin{tabular}{|c|c|c|c|c|c|c|c|c|c|}
\hline Datasets & Methods & $A c c \uparrow$ & Sub_Acc $\uparrow$ & F-score $\uparrow$ & H_Loss $\downarrow$ & Avg_Prec $\uparrow$ & $\operatorname{Cov} \downarrow$ & O_Error $\downarrow$ & R_Loss $\downarrow$ \\
\hline \multirow[t]{3}{*}{ BIBTEX } & MLKNN & 0,9849 & 0,0152 & 0,1135 & 0,0151 & 0,1913 & 74,0838 & 0,7297 & 0,3122 \\
\hline & BagMLKNN & 0,9857 & 0,0156 & 0,1166 & 0,0143 & 0,2185 & 70,6692 & 0,7032 & 0,3045 \\
\hline & CobMLKNN & 0,9850 & 0,0174 & 0,1338 & 0,0150 & 0,2421 & 75,3271 & 0,7860 & 0,2860 \\
\hline \multirow[t]{3}{*}{ COREL5K } & MLKNN & 0,9908 & 0,0000 & 0,0001 & 0,0092 & 0,2056 & 132,9772 & 0,7750 & 0,1834 \\
\hline & agMLKNN & 0,9908 & 0,0000 & 0,0071 & 0,0092 & 0,2176 & 131,1602 & 0,7558 & 0,1676 \\
\hline & CobMLKNN & 0,9901 & 0,0004 & 0,0434 & 0,0099 & 0,2074 & 139,6455 & 0,8120 & 0,1638 \\
\hline \multirow[t]{3}{*}{ ENRON } & MLKNN & 0,9368 & 0,0256 & 0,1444 & 0,0632 & 0,5235 & 15,7520 & 0,4607 & 0,1212 \\
\hline & BagMLKNN & 0,9382 & 0,0462 & 0,3575 & 0,0618 & 0,5448 & 16,6339 & 0,4568 & 0,1282 \\
\hline & CobMLKNN & 0,9435 & 0,0123 & 0,3846 & 0,0565 & 0,5591 & 15,6247 & 0,3944 & 0,1183 \\
\hline \multirow[t]{3}{*}{ EMOTIONS } & MLKNN & 0,6414 & 0,0475 & 0,2071 & 0,3586 & 0,5560 & 3,1535 & 0,6202 & 0,4465 \\
\hline & BagMLKNN & 0,6689 & 0,0515 & 0,2647 & 0,3311 & 0,5808 & 3,0576 & 0,5758 & 0,4312 \\
\hline & CobMLKNN & 0,6764 & 0,0606 & 0,2519 & 0,3236 & 0,5706 & 3,1495 & 0,5616 & 0,4176 \\
\hline \multirow[t]{3}{*}{ FLAGS } & MLKNN & 0,6040 & 0,0492 & 0,5629 & 0,3960 & 0,7401 & 4,0585 & 0,3050 & 0,2964 \\
\hline & BagMLKNN & 0,6176 & 0,0431 & 0,5964 & 0,3824 & 0,7475 & 4,0062 & 0,3018 & 0,2846 \\
\hline & CobMLKNN & 0,6369 & 0,0554 & 0,5957 & 0,3631 & 0,7615 & 3,9938 & 0,2862 & 0,2782 \\
\hline \multirow[t]{3}{*}{ FOOD } & MLKNN & 0,8225 & 0,1279 & 0,4284 & 0,1775 & 0,6880 & 3,8618 & 0,3471 & 0,1878 \\
\hline & BagMLKNN & 0,8359 & 0,1941 & 0,4484 & 0,1641 & 0,7164 & 3,6618 & 0,3044 & 0,1710 \\
\hline & CobMLKNN & 0,8446 & 0,2368 & 0,4531 & 0,1554 & 0,7264 & 3,6824 & 0,2882 & 0,1691 \\
\hline \multirow[t]{3}{*}{ GENEBASE } & MLKNN & 0,9694 & 0,3864 & 0,5041 & 0,0306 & 0,9345 & 1,4009 & 0,0751 & 0,0329 \\
\hline & BagMLKNN & 0,9787 & 0,6262 & 0,7096 & 0,0213 & 0,9048 & 2,4109 & 0,0851 & 0,0612 \\
\hline & CobMLKNN & 0,9821 & 0,6977 & 0,7684 & 0,0179 & 0,9570 & 1,5312 & 0,0226 & 0,0289 \\
\hline \multirow[t]{3}{*}{ MEDICAL } & MLKNN & 0,9724 & 0,1737 & 0,3023 & 0,0276 & 0,5687 & 6,4043 & 0,5368 & 0,1303 \\
\hline & BagMLKNN & 0,9747 & 0,1650 & 0,3246 & 0,0253 & 0,5615 & 7,0417 & 0,5485 & 0,1737 \\
\hline & CobMLKNN & 0,9764 & 0,1859 & 0,3571 & 0,0236 & 0,6396 & 5,6699 & 0,4466 & 0,1153 \\
\hline \multirow[t]{3}{*}{ SCENE } & MLKNN & 0,8573 & 0,3785 & 0,5269 & 0,1426 & 0,7706 & 0,7730 & 0,3820 & 0,1369 \\
\hline & BagMLKNN & 0,8672 & 0,3960 & 0,5144 & 0,1468 & 0,7306 & 1,0451 & 0,4201 & 0,1899 \\
\hline & CobMLKNN & 0,8791 & 0,4443 & 0,5940 & 0,1208 & 0,8095 & 0,6356 & 0,3211 & 0,1100 \\
\hline \multirow[t]{3}{*}{ YEAST } & MLKNN & 0,7710 & 0,0891 & 0,5750 & 0,2290 & 0,7105 & 6,9117 & 0,3015 & 0,2066 \\
\hline & BagMLKNN & 0,7591 & 0,1025 & 0,5572 & 0,2409 & 0,6989 & 7,0757 & 0,3007 & 0,2175 \\
\hline & CobMLKNN & 0,7846 & 0,1136 & 0,5911 & 0,2154 & 0,7228 & 6,7963 & 0,2665 & 0,1965 \\
\hline Averaged & MLKNN & 2.75 & 2.65 & 2.8 & 2.65 & 2.6 & 2.2 & 2.5 & 2.5 \\
\hline \multirow[t]{2}{*}{ Ranking } & BagMLKNN & 1.95 & 2.15 & 2 & 2.05 & 2.2 & 2.1 & 2.1 & 2.5 \\
\hline & CobMLKNN & 1.3 & 1.2 & 1.2 & 1.3 & 1.2 & 1.7 & 1.4 & 1 \\
\hline
\end{tabular}

\section{F-score}

The F-score metric uses both precision and recall, which is computed as the harmonic mean of the two measures. Precision is the proportion of labels correctly classified of the predicted positive labels, averaged over all instances. However, recall is the fraction of predicted correct labels of the actual labels.

From the results, it is noted that CobMLKNN achieves better scores than BagMLKNN and MLKNN. Thus, a high proportion of the predicted labels by CobMLKNN are relevant, although, many other labels are not correctly predicted by the classifier, especially in the extreme case with 10 and 30\% labeled rate. Indeed, in the Flags dataset (Tables 2 and 3), BagMLKNN approach generalizes better than
CobMLKNN, this can be explained by a very small number of instances $(10 \%=19)$ and the highest label density for the Flags dataset $L_{D}=0.485$ (Table 1).

\section{Hamming Loss}

Hamming Loss (H_Loss) is a commonly used evaluation metric in Multi-label classification. It represents the fraction of the wrong labels to the total number of labels.

This is the main reason for the low value of HammingLoss for almost all databases with the different labeling thresholds (see Tables 2,3,4 and 5). Except in the case of the $10 \%$ threshold, we note that CobMLKNN achieved the best performances on datasets with a high number of la- 
Table 4: Results under a labeled rate $\mu=40 \%$

\begin{tabular}{|c|c|c|c|c|c|c|c|c|c|}
\hline Datasets & Methods & $\operatorname{Acc} \uparrow$ & Sub_Acc $\uparrow$ & F-score $\uparrow$ & H_Loss $\downarrow$ & Avg_Prec $\uparrow$ & $\operatorname{Cov} \downarrow$ & O_Error $\downarrow$ & R_Loss $\downarrow$ \\
\hline \multirow[t]{3}{*}{ BIBTEX } & MLKNN & 0,9859 & 0,0237 & 0,1475 & 0,0151 & 0,2114 & 67,5643 & 0,6779 & 0,3012 \\
\hline & BagMLKNN & 0,9849 & 0,0162 & 0,1476 & 0,0147 & 0,2354 & 71,4294 & 0,7203 & 0,2947 \\
\hline & CobMLKNN & 0,9853 & 0,0276 & 0,1479 & 0,0141 & 0,2655 & 72,3009 & 0,7506 & 0,2736 \\
\hline \multirow[t]{3}{*}{ COREL5K } & MLKNN & 0,9908 & 0,0000 & 0,0001 & 0,0092 & 0,2193 & 128,9945 & 0,7724 & 0,1683 \\
\hline & BagMLKNN & 0,9908 & 0,0001 & 0,0106 & 0,0092 & 0,2299 & 127,4991 & 0,7544 & 0,1579 \\
\hline & CobMLKNN & 0,9902 & 0,0011 & 0,0536 & 0,0098 & 0,2190 & 134,0126 & 0,7928 & 0,1551 \\
\hline \multirow[t]{3}{*}{ ENRON } & MLKNN & 0,9372 & 0,0353 & 0,2899 & 0,0628 & 0,5237 & 15,1764 & 0,4818 & 0,1148 \\
\hline & BagMLKNN & 0,9419 & 0,0395 & 0,3789 & 0,0597 & 0,5431 & 15,0201 & 0,4279 & 0,1163 \\
\hline & CobMLKNN & 0,9403 & 0,0589 & 0,3772 & 0,0559 & 0,5892 & 15,9386 & 0,3587 & 0,1139 \\
\hline \multirow[t]{3}{*}{ EMOTIONS } & MLKNN & 0,6663 & 0,0586 & 0,3113 & 0,3337 & 0,5856 & 3,0455 & 0,5606 & 0,4163 \\
\hline & BagMLKNN & 0,6714 & 0,0586 & 0,2657 & 0,3286 & 0,5972 & 2,9404 & 0,5606 & 0,3936 \\
\hline & CobMLKNN & 0,6721 & 0,0576 & 0,2672 & 0,3279 & 0,5937 & 2,9616 & 0,5535 & 0,3921 \\
\hline \multirow[t]{3}{*}{ FLAGS } & MLKNN & 0,6382 & 0,0431 & 0,6183 & 0,3468 & 0,7841 & 4,0400 & 0,2472 & 0,2597 \\
\hline & BagMLKNN & 0,6432 & 0,0523 & 0,6224 & 0,3556 & 0,7861 & 3,9508 & 0,2694 & 0,2530 \\
\hline & CobMLKNN & 0,6475 & 0,0554 & 0,6415 & 0,3552 & 0,7965 & 3,9846 & 0,2134 & 0,2509 \\
\hline \multirow[t]{3}{*}{ FOOD } & MLKNN & 0,8210 & 0,1456 & 0,4430 & 0,1790 & 0,6946 & 3,9706 & 0,3500 & 0,1847 \\
\hline & BagMLKNN & 0,8276 & 0,1647 & 0,4429 & 0,1724 & 0,7206 & 3,9662 & 0,3015 & 0,1797 \\
\hline & CobMLKNN & 0,8431 & 0,2500 & 0,4571 & 0,1569 & 0,7386 & 3,9044 & 0,2559 & 0,1749 \\
\hline \multirow[t]{3}{*}{ GENEBASE } & MLKNN & 0,9683 & 0,3855 & 0,5055 & 0,0317 & 0,9179 & 1,6887 & 0,0643 & 0,0482 \\
\hline & BagMLKNN & 0,9854 & 0,7475 & 0,7904 & 0,0167 & 0,9353 & 1,4326 & 0,0653 & 0,0395 \\
\hline & CobMLKNN & 0,9883 & 0,7903 & 0,8209 & 0,0146 & 0,9595 & 2,0208 & 0,0624 & 0,0285 \\
\hline \multirow[t]{3}{*}{ MEDICAL } & MLKNN & 0,9729 & 0,1248 & 0,3723 & 0,0271 & 0,5793 & 5,8411 & 0,5301 & 0,1225 \\
\hline & BagMLKNN & 0,9756 & 0,1902 & 0,3464 & 0,0244 & 0,5616 & 6,0092 & 0,5466 & 0,1319 \\
\hline & CobMLKNN & 0,9782 & 0,2270 & 0,4136 & 0,0218 & 0,6411 & 5,1828 & 0,4491 & 0,1067 \\
\hline \multirow[t]{3}{*}{ SCENE } & MLKNN & 0,8832 & 0,4827 & 0,6173 & 0,1167 & 0,8149 & 0,6236 & 0,3104 & 0,1078 \\
\hline & BagMLKNN & 0,8857 & 0,5476 & 0,6505 & 0,1142 & 0,8029 & 0,7266 & 0,3197 & 0,1278 \\
\hline & CobMLKNN & 0,8992 & 0,5847 & 0,6863 & 0,1007 & 0,8334 & 0,6177 & 0,2688 & 0,1054 \\
\hline \multirow[t]{3}{*}{ YEAST } & MLKNN & 0,7862 & 0,1060 & 0,5966 & 0,2138 & 0,7355 & 6,6933 & 0,2558 & 0,1886 \\
\hline & BagMLKNN & 0,7766 & 0,0916 & 0,5768 & 0,2234 & 0,7202 & 6,9772 & 0,2764 & 0,2035 \\
\hline & CobMLKNN & 0,7889 & 0,1223 & 0,5979 & 0,2111 & 0,7316 & 6,6759 & 0,2543 & 0,1921 \\
\hline \multirow{3}{*}{$\begin{array}{l}\text { Averaged } \\
\text { Ranking }\end{array}$} & MLKNN & 2.55 & 2.65 & 2.5 & 2.55 & 2.5 & 2.2 & 2.15 & 2.5 \\
\hline & BagMLKNN & 2.05 & 2.15 & 2.3 & 2.15 & 2.1 & 1.8 & 2.45 & 2.4 \\
\hline & CobMLKNN & 1.4 & 1.2 & 1.2 & 1.3 & 1.4 & 2 & 1.4 & 1.1 \\
\hline
\end{tabular}

bels as Corel5K and Bibtex which have respectively 374 and 159 labels (Table 1), while for Flags, Emotions Scene with only seven and six labels, MLKNN performs better. These results are explained by the fact that this metric evaluates the frequency that labels in the Multi-label are misclassified i.e. It counts the number of labels for which our prediction is wrong and normalizes it.

\section{Average Precision}

The average precision is the average fraction of the labels ranked above a current label $y \in Y_{i}$ and which are actually in $Y_{i}$. The performance is perfect when avg_prec $=1$; the greater the value of avg_prec, the better is the performance.

According to avg_prec metric (Tables 2, 3, 4 and 5), CobMLKNN is always the most efficient for all sizes of learning sets except for the emotions dataset; characterized by the smallest number of labels and examples in the benchmark, the performance is very similar to those obtained by BagMLKNN algorithm.

\section{Coverage}

Coverage is used as an evaluation metric for multi-label example-based ranking and evaluates how many steps are needed, on average, to move down the ranked label list 
to cover all relevant labels of the example. For coverage, the smaller the metric value the better the system's performance.

The performances recorded for this metric through the different label thresholds (Tables 2,3,4 and 5) show that CobMLKNN is not always the best technique that achieves the minimum number of steps needed for a positive label to top the list. However, the Averaged Ranking indicates that this slight difference in performance is not statistically significant and put as the best method over BagMLKNN and MLKNN.

\section{One_error}

O_error evaluates how many times the first-order label is not in the set of relevant labels in the example. The metric one_error takes values between 0 and 1 . The lower the value of $\mathrm{O}$ error, the better the performance.

As we can see in Tables 2, 3, 4 and 5, CobMLKNN outperforms the other two classifiers. However, the provided results were poor for Bibtex and Corel5k datasets that are characterized by an important number of labels and a significant cardinality index, indicating important associativity of the labels with the examples of the datasets.

\section{Ranking Loss}

R_Loss evaluates the average fraction of reversely ordered label pairs of each instance. Overall results, we note that CobMLKNN achieves better results than BagMLKNN and $M L K N N$, especially, for datasets with a small number of instances of learning. However, in some cases, MLKNN was more effective for a large dataset. We remark also in Table 5 , for datasets with a small number of attributes, the two Ensemble methods do not generalize better than the $M L K N N$ classifier.

In summary, the predictive performance of the proposed method significantly improves when the number of learning examples increases, particularly when the size of the training set is greater than eight instances. Note that it is not surprising that for some data sets, all classifiers obtain very close performances for small learning sets; the number of examples provided is simply not enough to allow them to learn well. Moreover, the differences between the performance of CobMLKNN in comparison with the BagMLKNN and MLKNN classifiers increase linearly with the number of examples. In the next section, we compare the performances of all classifiers based on one vs. all com- parison, using Friedman's statistical tests (with a significance level of 5\%) for all measures.

\section{The post-hoc Friedman test}

To better assess the obtained results by each classifier previously, we adopt in this study the post-hoc Friedman test methodology proposed by Demsar [64] for the comparison of several algorithms over multiple datasets. The non-parametric Friedman test (free distribution) is used to compare observations repeated on the same subjects. When all classifiers are compared with a control classifier, to confirm the performance of each classifier, we calculated the $p$-value between two classifiers based on the Bonferroni procedure [65] which is a generally conservative, more flexible and easy test to use [64]. The p-value indicates whether a statistical hypothesis test is significant or not, and also indicates how much the performance is similar or different. When the p-value for this test is small (usually lower than 0.05), we have evidence to reject the null hypothesis.

The statistical tests for accuracy are shown in Table 6, the CobMLKNN is ranked first in the obtained ranking (i) for all labeled rate. The CobMLKNN vs. BagMLKNN gives a p-value of 0.1461 in 20, 30 and $40 \%$ labeled rate, which means a stability of performances for the different labeled rates. The $M L K N N$ is ranked third.

Regarding the Sub_Acc (Table 7) and the F-score (Table 8) results, we notice that the CobMLKNN is at the top for all labeled rate. The obtained $\mathrm{p}$-values indicate that CobM$L K N N$ results in a different and better performance compared to BagMLKNN and MLKNN.The same observations are noticed for the Hamming Loss metric (Table 9). Moreover, from Avg_Prec (Table 10), we can notice that CobM$L K N N$ is better than the two other algorithms: BagMLKNN and $M L K N N$, with only a small similarity for $80 \%$ labeled rate. The same remark can be observed for O_Error (Table 12) and R_Loss (Table 13) metric for a high labeled rate. Furthermore, Coverage's statistical tests (Table 11) result in very similar performances ( $p$-value $>0.3$ ), particularly with a labeling threshold of $40 \%$, this means that the convergence of CobMLKNN is similar to that of BagMLKNN and $M L K N N$, this similarity does not affect CobMLKNN performance.

In summary, it can be seen from Friedman's statistical tests, that CobMLKNN significantly outperforms BagM$L K N N$ and $M L K N N$ in case of a small number of labeled data with a high number of labels. This verifies the effectiveness of the collaborative approach for the Bagging $M L K N N$ in semi-supervised learning. 
Table 5: Results under a labeled rate $\mu=80 \%$

\begin{tabular}{|c|c|c|c|c|c|c|c|c|c|}
\hline Datasets & Methods & $\operatorname{Acc} \uparrow$ & Sub_Acc $\uparrow$ & F-score $\uparrow$ & H_Loss $\downarrow$ & Avg_Prec $\uparrow$ & $\operatorname{Cov} \downarrow$ & O_Error $\downarrow$ & R_Loss $\downarrow$ \\
\hline \multirow[t]{3}{*}{ BIBTEX } & MLKNN & 0,9849 & 0,0219 & 0,0055 & 0,0151 & 0,2761 & 66,0118 & 0,6741 & 0,2631 \\
\hline & BagMLKNN & 0,9851 & 0,0293 & 0,1590 & 0,0149 & 0,2298 & 68,3882 & 0,6532 & 0,2787 \\
\hline & CobMLKNN & 0,9860 & 0,0314 & 0,1635 & 0,0140 & 0,2941 & 62,2142 & 0,7258 & 0,2449 \\
\hline \multirow[t]{3}{*}{ COREL5K } & MLKNN & 0,9908 & 0,0000 & 0,0003 & 0,0092 & 0,2332 & 119,3650 & 0,7526 & 0,1425 \\
\hline & BagMLKNN & 0,9908 & 0,0008 & 0,0138 & 0,0092 & 0,2422 & 123,2125 & 0,7413 & 0,1500 \\
\hline & CobMLKNN & 0,9901 & 0,0012 & 0,0615 & 0,0099 & 0,2036 & 118,0809 & 0,8074 & 0,1403 \\
\hline \multirow[t]{3}{*}{ ENRON } & MLKNN & 0,9386 & 0,0271 & 0,2639 & 0,0614 & 0,5423 & 14,4963 & 0,4575 & 0,1067 \\
\hline & BagMLKNN & 0,9446 & 0,0339 & 0,4055 & 0,0587 & 0,5942 & 14,4099 & 0,4011 & 0,1043 \\
\hline & CobMLKNN & 0,9413 & 0,0342 & 0,4182 & 0,0554 & 0,5596 & 15,2568 & 0,3379 & 0,1135 \\
\hline \multirow[t]{3}{*}{ EMOTIONS } & MLKNN & 0,6835 & 0,0747 & 0,3526 & 0,3165 & 0,6203 & 0,7970 & 0,5061 & 0,3707 \\
\hline & BagMLKNN & 0,7113 & 0,0788 & 0,3409 & 0,2887 & 0,6651 & 2,5788 & 0,4606 & 0,3150 \\
\hline & CobMLKNN & 0,7141 & 0,0909 & 0,3606 & 0,2859 & 0,6588 & 2,5879 & 0,4444 & 0,3179 \\
\hline \multirow[t]{3}{*}{ FLAGS } & MLKNN & 0,6470 & 0,0585 & 0,6032 & 0,3587 & 0,7692 & 4,1754 & 0,3068 & 0,2837 \\
\hline & BagMLKNN & 0,6514 & 0,0615 & 0,6156 & 0,3565 & 0,7655 & 4,1815 & 0,2602 & 0,2851 \\
\hline & CobMLKNN & 0,6563 & 0,0523 & 0,6175 & 0,3543 & 0,7515 & 4,2400 & 0,2510 & 0,2960 \\
\hline \multirow[t]{3}{*}{ FOOD } & MLKNN & 0,8374 & 0,1794 & 0,4485 & 0,1626 & 0,7048 & 3,9676 & 0,3191 & 0,1841 \\
\hline & BagMLKNN & 0,8418 & 0,2265 & 0,4506 & 0,1582 & 0,7272 & 3,9941 & 0,2824 & 0,1817 \\
\hline & CobMLKNN & 0,8510 & 0,3015 & 0,4501 & 0,1490 & 0,7339 & 3,8647 & 0,2676 & 0,1748 \\
\hline \multirow[t]{3}{*}{ GENEBASE } & MLKNN & 0,9820 & 0,6824 & 0,7578 & 0,0180 & 0,9366 & 1,4697 & 0,0688 & 0,0299 \\
\hline & BagMLKNN & 0,9925 & 0,8715 & 0,9142 & 0,0075 & 0,9695 & 1,0833 & 0,0235 & 0,0188 \\
\hline & CobMLKNN & 0,9943 & 0,8914 & 0,9353 & 0,0057 & 0,9779 & 0,8407 & 0,0190 & 0,0127 \\
\hline \multirow[t]{3}{*}{ MEDICAL } & MLKNN & 0,9723 & 0,2270 & 0,3845 & 0,0277 & 0,6945 & 4,4748 & 0,3926 & 0,0773 \\
\hline & BagMLKNN & 0,9765 & 0,2528 & 0,4415 & 0,0235 & 0,6569 & 5,0730 & 0,4374 & 0,0917 \\
\hline & CobMLKNN & 0,9792 & 0,2920 & 0,5014 & 0,0208 & 0,7250 & 4,1834 & 0,3491 & 0,0707 \\
\hline \multirow[t]{3}{*}{ SCENE } & MLKNN & 0,8788 & 0,4982 & 0,6204 & 0,1211 & 0,7866 & 0,8049 & 0,3369 & 0,1433 \\
\hline & BagMLKNN & 0,8839 & 0,5194 & 0,6233 & 0,1160 & 0,8138 & 0,6528 & 0,3079 & 0,1124 \\
\hline & CobMLKNN & 0,8973 & 0,5501 & 0,6741 & 0,1026 & 0,8413 & 0,5391 & 0,2693 & 0,0902 \\
\hline \multirow[t]{3}{*}{ YEAST } & MLKNN & 0,7925 & 0,1385 & 0,6062 & 0,2075 & 0,7380 & 6,5159 & 0,2593 & 0,1847 \\
\hline & BagMLKNN & 0,7838 & 0,1236 & 0,6059 & 0,2162 & 0,7291 & 6,8025 & 0,2732 & 0,1941 \\
\hline & CobMLKNN & 0,7964 & 0,1285 & 0,6157 & 0,2036 & 0,7519 & 6,4516 & 0,2427 & 0,1748 \\
\hline \multirow{3}{*}{$\begin{array}{l}\text { Averaged } \\
\text { Ranking }\end{array}$} & MLKNN & 2.75 & 2.7 & 2.8 & 2.75 & 2.4 & 2 & 2.6 & 2.3 \\
\hline & BagMLKNN & 1.95 & 2 & 2.1 & 2.05 & 2 & 2.4 & 2 & 2.2 \\
\hline & CobMLKNN & 1.3 & 1.3 & 1.1 & 1.2 & 1.6 & 1.6 & 1.4 & 1.5 \\
\hline
\end{tabular}


Table 6: Adjusted $p$-values (Acc)

\begin{tabular}{crrc}
\hline$\mu$ & $\mathrm{i}$ & algorithm & unadjusted $p$ \\
\hline $10 \%$ & 1 & CobMLKNN & - \\
& 2 & CobMLKNN vs. BagMLKNN & 0.044171 \\
& 3 & CobMLKNN vs. MLKNN & 0.000796 \\
\hline $30 \%$ & 1 & CobMLKNN & - \\
& 2 & CobMLKNN vs. BagMLKNN & 0.1461 \\
& 3 & CobMLKNN vs. MLKNN & 0.001186 \\
\hline $40 \%$ & 1 & CobMLKNN & - \\
& 2 & CobMLKNN vs. BagMLKNN & 0.1461 \\
& 3 & CobMLKNN vs. MLKNN & 0.010127 \\
\hline $80 \%$ & 1 & CobMLKNN & - \\
& 2 & CobMLKNN vs. BagMLKNN & 0.1461 \\
& 3 & CobMLKNN vs. MLKNN & 0.001186 \\
\hline
\end{tabular}

Table 7: Adjusted $p$-values (Sub_Acc)

\begin{tabular}{crrc}
\hline$\mu$ & $\mathrm{i}$ & algorithm & unadjusted $p$ \\
\hline $10 \%$ & 1 & CobMLKNN & - \\
& 2 & CobMLKNN vs. BagMLKNN & 0.057347 \\
& 3 & CobMLKNN vs. MLKNN & 0.013906 \\
\hline $30 \%$ & 1 & CobMLKNN & - \\
& 2 & CobMLKNN vs. BagMLKNN & 0.033648 \\
& 3 & CobMLKNN vs. MLKNN & 0.001186 \\
\hline $40 \%$ & 1 & CobMLKNN & - \\
& 2 & CobMLKNN vs. BagMLKNN & 0.033648 \\
& 3 & CobMLKNN vs. MLKNN & 0.001186 \\
\hline $80 \%$ & 1 & CobMLKNN & - \\
& 2 & CobMLKNN vs. BagMLKNN & 0.117525 \\
& 3 & CobMLKNN vs. MLKNN & 0.001745 \\
\hline
\end{tabular}

Table 8: Adjusted $p$-values (F-score)

\begin{tabular}{crrc}
\hline$\mu$ & $\mathrm{i}$ & algorithm & unadjusted $p$ \\
\hline $10 \%$ & 1 & CobMLKNN & - \\
& 2 & CobMLKNN vs. BagMLKNN & 0.044171 \\
& 3 & CobMLKNN vs. MLKNN & 0.00729 \\
\hline $30 \%$ & 1 & CobMLKNN & - \\
& 2 & CobMLKNN vs. BagMLKNN & 0.073638 \\
& 3 & CobMLKNN vs. MLKNN & 0.000347 \\
\hline $40 \%$ & 1 & CobMLKNN & - \\
& 2 & CobMLKNN vs. BagMLKNN & 0.013906 \\
& 3 & CobMLKNN vs. MLKNN & 0.00365 \\
\hline $80 \%$ & 1 & CobMLKNN & - \\
& 2 & CobMLKNN vs. BagMLKNN & 0.025347 \\
& 3 & CobMLKNN vs. MLKNN & 0.000144 \\
\hline
\end{tabular}

Table 9: Adjusted $p$-values (H_Loss)

\begin{tabular}{crrc}
\hline$\mu$ & $\mathrm{i}$ & algorithm & unadjusted $p$ \\
\hline $10 \%$ & 1 & CobMLKNN & - \\
& 2 & CobMLKNN vs. MLKNN & 0.057347 \\
& 3 & CobMLKNN vs. BagMLKNN & 0.013906 \\
\hline $30 \%$ & 1 & CobMLKNN & - \\
& 2 & CobMLKNN vs. BagMLKNN & 0.093533 \\
& 3 & CobMLKNN vs. MLKNN & 0.002539 \\
\hline $40 \%$ & 1 & CobMLKNN & - \\
& 2 & CobMLKNN vs. BagMLKNN & 0.057347 \\
& 3 & CobMLKNN vs. MLKNN & 0.005189 \\
\hline $80 \%$ & 1 & CobMLKNN & - \\
& 2 & CobMLKNN vs. BagMLKNN & 0.057347 \\
& 3 & CobMLKNN vs. MLKNN & 0.000528 \\
\hline
\end{tabular}

Table 10: Adjusted $p$-values (Avg_Prec)

\begin{tabular}{crrc}
\hline$\mu$ & $\mathrm{i}$ & algorithm & unadjusted $p$ \\
\hline $10 \%$ & 1 & CobMLKNN & - \\
& 2 & CobMLKNN vs. MLKNN & 0.013906 \\
& 3 & CobMLKNN vs. BagMLKNN & 0.00365 \\
\hline $30 \%$ & 1 & CobMLKNN & - \\
& 2 & CobMLKNN vs. BagMLKNN & 0.025347 \\
& 3 & CobMLKNN vs. MLKNN & 0.001745 \\
\hline $40 \%$ & 1 & CobMLKNN & - \\
& 2 & CobMLKNN vs. BagMLKNN & 0.117525 \\
& 3 & CobMLKNN vs. MLKNN & 0.013906 \\
\hline $80 \%$ & 1 & CobMLKNN & - \\
& 2 & CobMLKNN vs. BagMLKNN & 0.371093 \\
& 3 & CobMLKNN vs. MLKNN & 0.073638 \\
\hline
\end{tabular}

Table 11: Adjusted $p$-values (Cov)

\begin{tabular}{crrc}
\hline$\mu$ & $\mathrm{i}$ & algorithm & unadjusted $p$ \\
\hline $10 \%$ & 1 & CobMLKNN & - \\
& 2 & CobMLKNN vs. BagMLKNN & 0.371093 \\
& 3 & CobMLKNN vs. MLKNN & 0.073638 \\
\hline $30 \%$ & 1 & CobMLKNN & - \\
& 2 & CobMLKNN vs. BagMLKNN & 0.371093 \\
& 3 & CobMLKNN vs. MLKNN & 0.263552 \\
\hline $40 \%$ & 1 & BagMLKNN & - \\
& 2 & BagMLKNN vs. CobMLKNN & 0.654721 \\
& 3 & BagMLKNN vs. MLKNN & 0.371093 \\
\hline $80 \%$ & 1 & CobMLKNN & - \\
& 2 & CobMLKNN vs. MLKNN & 0.371093 \\
& 3 & CobMLKNN vs. BagMLKNN & 0.073638 \\
\hline
\end{tabular}


Table 12: Adjusted $p$-values (O_Error)

\begin{tabular}{crrc}
\hline$\mu$ & $\mathrm{i}$ & algorithm & unadjusted $p$ \\
\hline $10 \%$ & 1 & CobMLKNN & - \\
& 2 & CobMLKNN vs. BagMLKNN & 0.073638 \\
& 3 & CobMLKNN vs. MLKNN & 0.00365 \\
\hline $30 \%$ & 1 & CobMLKNN & - \\
& 2 & CobMLKNN vs. BagMLKNN & 0.117525 \\
& 3 & CobMLKNN vs. MLKNN & 0.013906 \\
\hline $40 \%$ & 1 & CobMLKNN & - \\
& 2 & CobMLKNN vs. MLKNN & 0.093533 \\
& 3 & CobMLKNN vs. BagMLKNN & 0.01888 \\
\hline $80 \%$ & 1 & CobMLKNN & - \\
& 2 & CobMLKNN vs. BagMLKNN & 0.179712 \\
& 3 & CobMLKNN vs. MLKNN & 0.00729 \\
\hline
\end{tabular}

Table 13: Adjusted $p$-values (R_Loss)

\begin{tabular}{crrc}
\hline$\mu$ & $\mathrm{i}$ & algorithm & unadjusted $p$ \\
\hline $10 \%$ & 1 & CobMLKNN & - \\
& 2 & CobMLKNN vs. BagMLKNN & 0.00365 \\
& 3 & CobMLKNN vs. MLKNN & 0.000144 \\
\hline $30 \%$ & 1 & CobMLKNN & - \\
& 2 & CobMLKNN vs. MLKNN & 0.000796 \\
& 2 & CobMLKNN vs. BagMLKNN & 0.000796 \\
\hline $40 \%$ & 1 & CobMLKNN & - \\
& 2 & CobMLKNN vs. BagMLKNN & 0.00365 \\
& 3 & CobMLKNN vs. MLKNN & 0.001745 \\
\hline $80 \%$ & 1 & CobMLKNN & - \\
& 2 & CobMLKNN vs. BagMLKNN & 0.117525 \\
& 3 & CobMLKNN vs. MLKNN & 0.073638 \\
\hline
\end{tabular}

\section{Conclusion}

In this work, we studied the problem of semi-supervised learning for a Multi-label framework. We proposed collaborative Multi-label learning using an ensemble learning named CobMLKNN.

We carried out an extensive experimental comparison between three algorithms named: MLKNN, BagMLKNN, and CobMLKNN, we used for that several evaluation measures on ten Multi-label datasets. The results obtained show that CobMLKNN gives very good predictive performances compared to other supervised classifiers regardless of the size of the subset of learning, due to the use of complementary Multi-label classifiers in the ensemble built based on newly labeled learning examples with degree confidence exceeding 85\%.
It is clear that semi-supervised learning guided by Ensemble methods leads to better results and that explain the interests of many researchers to address the semisupervised learning-based Ensemble methods in many fields. An interesting follow-up to our work would be to remove irrelevant and/or redundant features from the learning set that might be as a noise for the classifier that should be learned in a semi-supervised way, the use of some multivariate measures for that could be a good option for future works.

\section{References}

[1] Madjarov G., Kocev D., Gjorgjevikj D., Deroski S., An Extensive Experimental Comparison of Methods for Multilabel Learning, Pattern Recogn., 45(9), 2012, 3084-3104, DOI:10.1016/j.patcog.2012.03.004

[2] Zhang M.L., Zhou Z.H., ML-KNN: A Lazy Learning Approach to Multi-label Learning, Pattern Recogn., 40((7)), 2007, 20382048, DOI:10.1016/j.patcog.2006.12.019

[3] Blum A., Mitchell T., Combining labeled and unlabeled data with co-training, in Proceedings of the eleventh annual conference on Computational learning theory, COLT' 98 , New York, NY, USA, 1998, 92-100

[4] Li M., Zhou Z.H., Improve Computer-Aided Diagnosis With Machine Learning Techniques Using Undiagnosed Samples, IEEE Transactions on Systems, Man, and Cybernetics - Part A: Systems and Humans, 37(6), 2007, 1088-1098, DOI:10.1109/TSMCA.2007.904745

[5] Madjarov G., Kocev D., Gjorgjevikj D., Deroski S., An Extensive Experimental Comparison of Methods for Multilabel Learning, Pattern Recogn., 45(9), 2012, 3084-3104, DOI:10.1016/j.patcog.2012.03.004

[6] Herrera F., Charte F., Rivera A.J., del Jesús M.J., Multilabel Classification - Problem Analysis, Metrics and Techniques, Springer, 2016, DOI:10.1007/978-3-319-41111-8

[7] Schapire R.E., Singer Y., BoosTexter: A Boosting-based System for Text Categorization, Machine Learning, 39, 2000, 135-168

[8] Boutell M.R., Luo J., Shen X., Brown C.M., Learning multi-label scene classification, Pattern Recognition, 37(9), 2004, 17571771, DOI: 10.1016/j.patcog.2004.03.009

[9] Zhang D., Liu X., Ensemble of k-Labelset Classifiers for Multilabel Image Classification, in Z. Shi, D. Leake, S. Vadera, eds., Intelligent Information Processing VI, Springer Berlin Heidelberg, Berlin, Heidelberg, 2012, 364-371

[10] Liu Y., Sheng L., Shao J., Yan J., Xiang S., Pan C., Multi-Label Image Classification via Knowledge Distillation from WeaklySupervised Detection, in Proceedings of the 26th ACM International Conference on Multimedia, MM '18, ACM, New York, NY, USA, 2018, 700-708, DOI:10.1145/3240508.3240567

[11] Wang Q., Jia N., Breckon T.P., A Baseline for Multi-Label Image Classification Using Ensemble Deep CNN, Computing Research Repository (CoRR), 1811.08, 2018

[12] Guan Q., Huang Y., Multi-label chest X-ray image classification via category-wise residual attention learning, Pattern Recognition Letters, 2018, DOI:10.1016/j.patrec.2018.10.027 
[13] Oramas S., Nieto O., Barbieri F., Serra X., Multi-label Music Genre Classification from Audio, Text, and Images Using Deep Features, Computing Research Repository (CoRR), abs/1707.04916, 2017

[14] Huang K.H., Lin H.T., Cost-sensitive label embedding for multilabel classification, Machine Learning, 106(9), 2017, 1725-1746, DOI:10.1007/s10994-017-5659-z

[15] Sun L., Kudo M., Multi-label classification by polytreeaugmented classifier chains with label-dependent features, Pattern Analysis and Applications, 22(3), 2019, 1029-1049, DOI:10.1007/s10044-018-0711-6

[16] Snoek C.G.M., Worring M., van Gemert J.C., Geusebroek J.M., Smeulders A.W.M., The Challenge Problem for Automated Detection of 101 Semantic Concepts in Multimedia, in Proceedings of the 14th ACM International Conference on Multimedia, MM '06, ACM, New York, NY, USA, 2006, 421-430, DOI:10.1145/1180639.1180727

[17] Abu-El-Haija S., Kothari N., Lee J., Natsev P., Toderici G., Varadarajan B., Vijayanarasimhan S., YouTube-8M: A LargeScale Video Classification Benchmark, volume abs/1609.08675, 2016

[18] Na S., Yu Y., Lee S., Kim J., Kim G., Encoding Video and Label Priors for Multi-label Video Classification on YouTube-8M dataset, Computing Research Repository (CoRR), abs/1706.07960, 2017

[19] Shin K., Jeon J., Lee S., Lim B., Jeong M., Nang J., Approach for Video Classification with Multi-label on YouTube-8M Dataset, Computing Research Repository (CoRR), abs/1808.08671, 2018

[20] Rak R., Kurgan L., Reformat M., A Tree-projection-based Algorithm for Multi-label Recurrent-item Associative-classification Rule Generation, Data \& Knowledge Engineering, 64(1), 2008, 171-197, DOI:10.1016/j.datak.2007.05.006

[21] Ji S., Tang L., Yu S., Ye J., Extracting shared subspace for multilabel classification, in Proceedings of the ACM SIGKDD International Conference on Knowledge Discovery and Data Mining, 2008, 381-389, DOI:10.1145/1401890.1401939

[22] Nguyen L.T.T., Mining Class Association Rules with the Difference of Obidsets, in N.T. Nguyen, B. Attachoo, B. Trawiński, K. Somboonviwat, eds., Intelligent Information and Database Systems, Springer International Publishing, Cham, 2014, 72-81

[23] Tzima F.A., Allamanis M., Filotheou A., Mitkas P.A., Inducing Generalized Multi-Label Rules with Learning Classifier Systems, Computing Research Repository (CoRR), abs/1512.07982, 2015

[24] Eneldo L.M., Janssen F., Learning rules for multi-label classification: a stacking and a separate-and-conquer approach, Machine Learning, 105(1), 2016, 77-126, DOI:10.1007/s10994-016-55521

[25] Curi Z., de Souza Britto Jr. A., Paraiso E.C., Multi-label Classification of User Reactions in Online News, Computing Research Repository (CoRR), abs/1809.02811, 2018

[26] Katakis I., Vlahavas G.T.I., Multilabel text classification for automated tag suggestion, in Proceedings of the ECML/PKDD, volume 18, 2008

[27] Khan A.U.R., Khan M., Khan M.B., Khan A.U.R., Khan M., Khan M.B., Naïve Multi-label Classification of YouTube Comments Using Comparative Opinion Mining, Procedia Computer Science, 82, 2016, 57-64, DOI:10.1016/j.procs.2016.04.009, 4th Symposium on Data Mining Applications, SDMA2016, 30 March 2016, Riyadh, Saudi Arabia

[28] Yu K., Yu S., Tresp V., Multi-label Informed Latent Semantic Indexing, in Proceedings of the 28th Annual International ACM SI-
GIR Conference on Research and Development in Information Retrieval, SIGIR '05, ACM, New York, NY, USA, 2005, 258-265, DOI:10.1145/1076034.1076080

[29] Elghazel H., Aussem A., Gharroudi O., Saadaoui W., Ensemble multi-label text categorization based on rotation forest and latent semantic indexing, Expert Systems with Applications, 57, 2016, 1-11, DOI:10.1016/j.eswa.2016.03.041

[30] Papanikolaou Y., Tsoumakas G., Laliotis M., Markantonatos N., Vlahavas I., Large-scale online semantic indexing of biomedical articles via an ensemble of multi-label classification models, Journal of Biomedical Semantics, 8(1), 2017, 43, DOI:10.1186/s13326-017-0150-0

[31] Clare A., King R.D., Knowledge Discovery in Multi-label Phenotype Data., in L.D. Raedt, A. Siebes, eds., European Conference on Principles of Data Mining and Knowledge Discovery, volume 2168 of Lecture Notes in Computer Science, Springer, 2001, 4253

[32] Qiu W.R., Zheng Q.S., Sun B.Q., Xiao X., Multi-iPPseEvo: A Multilabel Classifier for Identifying Human Phosphorylated Proteins by Incorporating Evolutionary Information into Chou's General PseAAC via Grey System Theory, Molecular Informatics, 36(3), 2017, 16-85, DOI:10.1002/minf.201600085

[33] Lin W., Xu D., Imbalanced multi-label learning for identifying antimicrobial peptides and their functional types, Bioinformatics, 32(24), 2016, 3745-3752, DOI:10.1093/bioinformatics/btw560

[34] Yu Y., Deng H., Zhu X., Research of ddi based on multi-label conditional random field, BIO Web of Conferences, 8(01039), 2017, 10-39, DOI:10.1051/bioconf/20170801039

[35] Tsoumakas G., Katakis I., Vlahavas I.P., Random k-Labelsets for Multilabel Classification, IEEE Trans. Knowl. Data Eng., 23(7), 2011, 1079-1089, DOI:10.1109/TKDE.2010.164

[36] Read J., Pfahringer B., Holmes G., Frank E., Classifier Chains for Multi-label Classification, Machine learning, 85((3)), 2011, 333359, DOI:10.1007/s10994-011-5256-5

[37] Tsoumakas G., Katakis I., Vlahavas I., Mining Multi-label Data, Springer US, Boston, MA, 2010, 667-685, DOI:10.1007/978-0387-09823-4_34

[38] Tsoumakas G., Katakis I., Multi-Label Classification: An Overview, IJDWM, 3(3), 2007, 1-13, DOI:10.4018/jdwm.2007070101

[39] Li R., Liu W., Lin Y., Zhao H., Zhang C., An Ensemble Multilabel Classification for Disease Risk Prediction, Journal of healthcare engineering, 2017, 8051673, DOI:10.1155/2017/8051673

[40] Read J., Pfahringer B., Holmes G., Multi-label Classification Using Ensembles of Pruned Sets, in Proceedings of the 2008 Eighth IEEE International Conference on Data Mining, ICDM '08, IEEE Computer Society, Washington, DC, USA, 2008, 995-1000, DOI:10.1109/ICDM.2008.74

[41] Read J., Pfahringer B., Holmes G., Frank E., Classifier chains for multi-label classification, Machine learning, 85(3), 2011, 333, DOI:10.1007/s10994-011-5256-5

[42] Breiman L., Random Forests, Machine learning, 45(1), 2001, 532, DOI:10.1023/A:1010933404324

[43] Shi C., Kong X., Yu P.S., Wang B., Multi-label Ensemble Learning, in Proceedings of the 2011 European Conference on Machine Learning and Knowledge Discovery in Databases - Volume Part III, ECML PKDD'11, Springer-Verlag, Berlin, Heidelberg, 2011, 223-239

[44] Guo Y., Schuurmans D., Semi-supervised Multi-label Classification, in P.A. Flach, T. De Bie, N. Cristianini, eds., Machine Learn- 
ing and Knowledge Discovery in Databases, Springer Berlin Heidelberg, Berlin, Heidelberg, 2012, 355-370

[45] Jiang M.X..F.S..X., Multi-label learning with co-training based on semi-supervised regression, in Proceedings 2014 IEEE International Conference on Security, Pattern Analysis, and Cybernetics (SPAC), 2014

[46] Zhan W., Zhang M.L., Inductive Semi-supervised Multi-Label Learning with Co-Training, in Proceedings of the 23rd ACM SIGKDD International Conference on Knowledge Discovery and Data Mining, KDD '17, ACM, New York, NY, USA, 2017, 1305-1314, DOI:10.1145/3097983.3098141

[47] Feng S., Xu D., Transductive Multi-Instance Multi-Label learning algorithm with application to automatic image annotation, Expert Systems with Applications, 37(1), 2010, 661-670, DOI:10.1016/j.eswa.2009.06.111

[48] Kong X., Ng M.K., Zhou Z.H., Transductive Multilabel Learning via Label Set Propagation, IEEE Trans. on Knowl. and Data Eng., 25(3), 2013, 704-719, DOI:10.1109/TKDE.2011.141

[49] Fu Y., Yang Y., Hospedales T.M., Xiang T., Gong S., Transductive Multi-class and Multi-label Zero-shot Learning, Computing Research Repository (CoRR), abs/1503.07884, 2015

[50] Zha Z.J., Mei T., Wang J., Wang Z., Hua X.S., Graph-based semi-supervised learning with multiple labels, Journal of Visual Communication and Image Representation, 20(2), 2009, 97103, DOI:10.1016/j.jvcir.2008.11.009, special issue on Emerging Techniques for Multimedia Content Sharing, Search and Understanding

[51] Dharmadhikari S.C., Ingle M., Kulkarni P., Semi Supervised Learning Based Text Classification Model for Multi Label Paradigm, in V.V. Das, P. Elkafrawy, eds., Signal Processing and Information Technology, Springer International Publishing, Cham, 2014, 178-184

[52] Dong H., Li Y., Zhou Z., Learning From Semi-Supervised WeakLabel Data, in Thirty-Second AAAI Conference on Artificial Intelligence, AAAI Press, 2018, 2926-2933

[53] Soleimani H., Miller D.J., Semi-supervised Multi-Label Topic Models for Document Classification and Sentence Labeling, in Proceedings of the 25th ACM International on Conference on Information and Knowledge Management, CIKM '16, ACM, New York, NY, USA, 2016, 105-114, DOI:10.1145/2983323.2983752

[54] Sun F., Xu M., Jiang X., Robust Multi-label Image Classification with Semi-Supervised Learning and Active Learning, in X. He, S. Luo, D. Tao, C. Xu, J. Yang, M.A. Hasan, eds., Multimedia Modeling, Springer International Publishing, Cham, 2015, 512-523
[55] Fu S.L..Y., Robust multi-label semi-supervised classification, in IEEE International Conference on Big Data (Big Data), Boston, MA, USA, 2017

[56] Gharroudi O., Elghazel H., Aussem A., A Semi-Supervised Ensemble Approach for Multi-label Learning, in IEEE 16th International Conference on Data Mining Workshops (ICDMW), 2016, 1197-1204, DOI:10.1109/ICDMW.2016.0173

[57] Xu Y., Wang J., An S., Wei J., Ruan J., Semi-Supervised Multi-Label Feature Selection by Preserving Feature-Label Space Consistency, in Proceedings of the 27th ACM International Conference on Information and Knowledge Management, CIKM '18, ACM, New York, NY, USA, 2018, 783-792, DOI:10.1145/3269206.3271760

[58] Pham T.N., Nguyen V.Q., Tran V.H., Nguyen T.T., Ha Q.T., A semi-supervised multi-label classification framework with feature reduction and enrichment, Journal of Information and Telecommunication, 1(2), 2017, 141-154, DOI:10.1080/24751839.2017.1323486

[59] Douibi K., Settouti N., Chikh M.A., The Homogeneous Ensemble Methods for MLknn Algorithm, in Proceedings of the International Conference on Compute and Data Analysis, ICCDA '17, ACM, New York, NY, USA, 2017, 197-201, DOI:10.1145/3093241.3093262

[60] Breiman L., Bagging Predictors, Mach. Learn., 24(2), 1996, 123140, DOI:10.1023/A:1018054314350

[61] Bellal F., Elghazel H., Aussem A., A semi-supervised feature ranking method with ensemble learning, Pattern Recognition Letters, 33(10), 2012, 1426-1433, DOI:10.1016/j.patrec.2012.03.001

[62] Nigam K., Ghani R., Analyzing the Effectiveness and Applicability of Co-training, in Proceedings of the Ninth International Conference on Information and Knowledge Management, CIKM '00, ACM, New York, NY, USA, 2000, 86-93, DOI:10.1145/354756.354805

[63] Tsoumakas G., Spyromitros X., Vilcek J., Vlahavas I., Mulan: A java library for multi-label learning, Journal of Machine Learning Research, 12, 2011, 2411-2414

[64] Demšar J., Statistical Comparisons of Classifiers over Multiple Data Sets, Journal of Machine Learning Research, 7, 2006, 1-30

[65] Derrac J., García S., Molina D., Herrera F., A practical tutorial on the use of nonparametric statistical tests as a methodology for comparing evolutionary and swarm intelligence algorithms, Swarm and Evolutionary Computation, 1(1), 2011, 3-18 